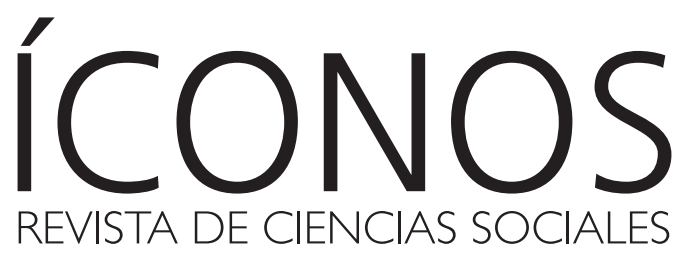

No. 61, Mayo 2018

ISSN 1390-1249

CDD 300.5 / CDU 3 / LC H8 .S8 F53

Vol. 22, Issue 2, May 2018

Quito, Ecuador

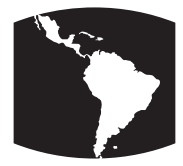

FLACSO

ECUADOR

Facultad Latinoamericana de Ciencias Sociales Sede Ecuador 
ÍCONOS. Revista de Ciencias Sociales

Número 61, mayo de 2018

Quito, Ecuador

ISSN: 1390-1249 / CDD: 300.5 / CDU: 3 / LC: H8 .S8 F53

(vol. 22, issue 2, mayo 2018)

Íconos. Revista de Ciencias Sociales es una publicación de FLACSO Ecuador. Fue fundada en 1997 con el fin de estimular una reflexión crítica desde las ciencias sociales sobre temas de debate social, político, cultural y económico del país, la región andina y América Latina en general. La revista está dirigida a la comunidad científica y a quienes se interesen por conocer, ampliar y profundizar, desde perspectivas académicas, estos temas. Íconos. Revista de Ciencias Sociales se publica cuatrimestralmente en los meses de enero, mayo y septiembre.

Íconos. Revista de Ciencias Sociales es parte de las siguientes bases, catálogos e índices:

Academic Search Premier Magazines and Journals EBSCOhost. Estados Unidos

CABELL'S. Directory of Publishing Opportunities. Estados Unidos

CIRC. Clasificación Integrada de Revistas Científicas. EC3metrics.

Universidad de Granada. España

CLASE. Citas Latinoamericanas en Ciencias Sociales. UNAM.

México

DIALNET. Universidad de la Rioja. Espańa

DOAJ. Directory of Open Access Journal. Lund University Libraries.

Suecia

ESCI. Emerging Source Citation Index. Web of Science. Clarivate Analytics

FLACSO Andes. FLACSO Ecuador

Fuente Académica Plus EBSCOhost. Estados Unidos

HAPI. Hispanic American Periodical Index. UCLA. Estados Unidos

IBSS. International Bibliography of the Social Science. ProQuest.

Estados Unidos

Informe Académico Thompson Gale. Estados Unidos

I2OR. International Institute of Organized Research. India, Australia LatAm-Studies. International Information Services. Estados Unidos

LATINDEX. Sistema Regional de Información en Línea para Revistas Científicas, de América Latina, el Caribe, España y Portugal. México

MIAR. Matriz de Información para el Análisis de Revistas. Universitat de Barcelona. España

Political Science Complete. EBSCOhost. Estados Unidos

REDALYC. Red de Revistas Científicas de América Latina y el Caribe, España y Portugal. UAEM. México

REDIB. Red Iberoamericana de Innovación y Conocimiento Científico. CSIC. España

SciELO. Scientific Electronic Library Online. Ecuador

Sociological Abstracts. CSA-ProQuest. Estados Unidos

Social Science Jornals. Sociology Collection. ProQuest. Estados Unidos

TIB. German National Library of Science and Technology. Alemania

Ulrich's Periodical Directory. CSA-ProQuest. Estados Unidos

WPSA. Worldwide Political Science Abstracts. ProQuest. Estados Unidos

Los artículos que se publican en la revista son de responsabilidad exclusiva de sus autores y autoras; no reflejan necesariamente el pensamiento de Íconos. Revista de Ciencias Sociales

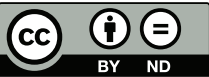

http://creativecommons.org/licenses/by-nd/3.0/deed.es

Director de FLACSO Ecuador: Juan Ponce

Directora de Íconos: Susana Wappenstein

Editora de Íconos: Jenny Pontón

Asistente editorial: Bárbara Sáez / Caroline Martínez

Correctora de estilo: Gabriela Chauvin

Traducción al inglés: Patrick Clark

Traducción al portugués: Javier Abi-Saab

Consejo editorial

Adrián Bonilla. Facultad Latinoamericana de Ciencias

Sociales. Ecuador

Víctor Bretón. Universitat de Lleida. España

Carolina Curvale. Facultad Latinoamericana de Ciencias

Sociales. Ecuador

Carmen Diana Deere. University of Florida. Estados Unidos

Hernán Ibarra. Centro Andino de Acción Popular. Ecuador

Catalina León. Universidad de Cuenca. Ecuador

Liisa North. York University. Canadá

Silvia Vega. Universidad Central del Ecuador

\section{Comité asesor internacional}

Javier Auyero. University of Texas, Austin. Estados Unidos

Bruce Bagley. University of Miami. Estados Unidos

Flavia Freidenberg. Universidad de Salamanca. España

Roberto Follari. Universidad Nacional de Cuyo. Argentina

Andrés Guerrero. Honorary Research Fellow. University

of Saint Andrews. Reino Unido

Magdalena León. Universidad Nacional. Colombia

Joan Martínez Alier. Universitat Autònoma de Barcelona. España

Carlos de Mattos. Pontificia Universidad Católica. Chile

Cecilia Méndez. University of California, Santa Bárbara.

Estados Unidos

Lorraine Nencel. Centro de Estudio y Documentación

Latinoamericana. Holanda

Joan Pujadas. Universitat Rovira i Virgili. España

Luca Queirolo. Università degli Studi di Genova. Italia

Francisco Rojas. University for Peace. Costa Rica

Rob Vos. International Institute of Social Studies. Holanda

Coordinadores del dossier "Geografías críticas en América Latina" Sofía Zaragocin Carvajal, Melissa Moreano Venegas, Soledad Álvarez Velasco

Imagen de portada: Sofía Acosta "La Suerte"

Diseño y diagramación: Antonio Mena / Shiti Rivadeneira Impresión: Editorial Ecuador

Envío de artículos, información, solicitud de canje:

revistaiconos@flacso.edu.ec

www.revistaiconos.ec

(CFLACSO Ecuador

Casilla: 17-11-06362

Dirección: Calle La Pradera E7-174 y Av. Diego de Almagro

Quito, Ecuador

Teléfonos: +593-2 294-6800 Fax: +593-2 294-6803

CDD 300.5, CDU 3, LC: H8 .S8 F53

Íconos: Revista de Ciencias Sociales. -Quito: FLACSO Ecuador, 1997-

v. : il. ; $28 \mathrm{~cm}$.

enero-abril 1997 -

Cuatrimestral- enero-mayo-septiembre

ISSN: $1390-1249$

1. Ciencias Sociales. 2. Ciencias Sociales-Ecuador. I. Facultad Latinoamericana de Ciencias Sociales (Ecuador) 
No. 61, Mayo 2018

ISSN 1390-1249

CDD 300.5 / CDU 3 / LC H8 .S8 F53

Vol. 22, Issue 2, May 2018

Quito, Ecuador

\section{Contenido}

Dossier

Hacia una reapropiación de la geografía crítica en América Latina

Presentación del dossier. . . . . . . . . . . . . . . . . . . . . . . . . 11-32

Sofía Zaragocin Carvajal, Melissa Moreano Venegas y Soledad Álvarez Velasco

Las "otras" geografías en América Latina: alternativas desde

los paisajes del pueblo Chatino

Gerónimo Barrera de la Torre

Geografías de la cocaína: trayectos de mujeres colombianas encarceladas por drogas en Ecuador

Ana María Cerón Cáceres

El mapa son los otros: narrativas del viaje de migrantes centroamericanos en la frontera sur de México

Rodrigo Parrini Roses y Edith Flores Pérez

Cartografía social de Chapiquiña: reivindicando los derechos territoriales indígenas en los Altos de Arica, Chile

Joselin Leal Landeros y Alan Rodríguez Valdivia

Ideologías geográficas y producción de la naturaleza: elementos para pensar la resignación de los bosques frente a la crisis del capital $115-133$

Luis Fernando De Matheus y Andrei Cornetta

Pueblo de papel: la producción social del territorio en el poblado industrial de Atenquique, México . $135-152$ Alejandro Ponce de León Pagaza 
Evocación a Jorge León Trujillo (1948-2017)

Hernán Ibarra

Ensayo visual

Cuerpo / territorio

$163-175$

Sofía Acosta "La Suerte"

Temas

Saber hablar: construcción del capital militante en movimientos

populares en Argentina

María Mercedes Palumbo

Prácticas políticas de los sectores populares en Río de Janeiro:

urbanización de la favela Santa Marta

Maximiliano Duarte Acquistapace

Reseñas

UNASUR: poder y acción en Suramérica

de Fabio Sánchez Cabarcas

César Augusto Niño González

The Crisis of Multiculturalism in Latin America

de David Lehmann, editor

Luis Fernando Gutiérrez Domínguez

Indígenas de la nación. Etnografía histórica de la alteridad

(Milpa Alta, siglos XVII-XXI)

de Paula López Caballero

$232-235$

Charlynne Curiel 
No. 61, Mayo 2018

ISSN 1390-1249

CDD 300.5 / CDU 3 / LC H8 .S8 F53

Vol. 22, Issue 2, May 2018

Quito, Ecuador

\section{Content}

Dossier

Towards a Re-appropriation of Critical Geography for Latin America Introduction to Dossier.

Sofía Zaragocin Carvajal, Melissa Moreano Venegas and Soledad Álvarez Velasco

The "Other" Geographies in Latin America: Alternatives from

the Landscapes of the Chatino People

Gerónimo Barrera de la Torre

Geographies of Cocaine: Trajectories of Colombian Women Imprisoned for Drug Trafficking in Ecuador

Ana María Cerón Cáceres

The Outsiders are the Map: Travel Narratives of Central American

Migrants on Mexico's Southern Border

Rodrigo Parrini Roses and Edith Flores Pérez

The Social Cartography of Chapiquiña: Revindicating Indigenous

Territorial Rights in the Highlands of Arica, Chile

Joselin Leal Landeros and Alan Rodríguez Valdivia

Geographical Ideologies and Nature: The Re-Making of Forests and the Crisis of Capital

Luis Fernando De Matheus and Andrei Cornetta

Paper Town: The Social Production of Territory in the Industrial Town

of Atenquique, Mexico

Alejandro Ponce de León Pagaza 
Remembering Jorge León Trujillo (1948-2017)

Hernán Ibarra

Visual essay

Body / Territory

$163-175$

Sofía Acosta "La Suerte"

Topics

Talking the Talk: The Construction of Activist Capital

in Argentinian Popular Social Movements

$179-202$

María Mercedes Palumbo

Political Practices of the Popular Sectors in Rio de Janeiro:

Urbanization of the Favela Santa Marta . . . . . . . . . . . . . . . . . . . . . 203-222

Maximiliano Duarte Acquistapace

Reviews

UNASUR: poder y acción en Suramérica

by Fabio Sánchez Cabarcas

César Augusto Niño González

The Crisis of Multiculturalism in Latin America

by David Lehmann, editor

Luis Fernando Gutiérrez Domínguez

Indígenas de la nación. Etnografía histórica de la alteridad

(Milpa Alta, siglos XVII-XXI)

by Paula López Caballero

$232-235$

Charlynne Curiel 
No. 61, Mayo 2018

ISSN 1390-1249

CDD 300.5 / CDU 3 / LC H8 .S8 F53

Vol. 22, Issue 2, May 2018

Quito, Ecuador

\section{Conteúdo}

Dossiê

Rumo a uma reapropriação de geografia crítica na América Latina

Apresentação do dossiê.

Sofía Zaragocin Carvajal, Melissa Moreano Venegas e Soledad Álvarez Velasco

As "outras" geografias na América Latina: alternativas desde as paisagens do povo Chatino

Gerónimo Barrera de la Torre

Geografias da cocaína: trajetos de mulheres colombianas encarceradas por drogas no Equador

Ana María Cerón Cáceres

O mapa são os outros: narrativas da viagem de migrantes

centro-americanos na fronteira sul do México

Rodrigo Parrini Roses e Edith Flores Pérez

Cartografia social de Chapiquiña: reivindicando os direitos territoriais

indígenas nos Altos de Arica, Chile

Joselin Leal Landeros e Alan Rodríguez Valdivia

Ideologias geográficas e natureza: a resignação dos bosques

na crise do capital

Luis Fernando De Matheus e Andrei Cornetta

Povoado de papel: a produção social do território no povoado industrial de Atenquique, no México

Alejandro Ponce de León Pagaza 
Evocação a Jorge León Trujillo (1948-2017).

Hernán Ibarra

Ensaio visual

Corpo / território $163-175$

Sofía Acosta "La Suerte"

Temas

Saber falar: construção do capital militante nos movimentos populares

na Argentina . . . . . . . . . . . . . . . . . . . . . . . . . . . . . 179-202

María Mercedes Palumbo

Práticas políticas dos setores populares no Rio de Janeiro:

urbanização da favela de Santa Marta

Maximiliano Duarte Acquistapace

Resenhas

UNASUR: poder y acción en Suramérica

de Fabio Sánchez Cabarcas

César Augusto Niño González

The Crisis of Multiculturalism in Latin America

de David Lehmann, editor

Luis Fernando Gutiérrez Domínguez

Indígenas de la nación. Etnografía histórica de la alteridad

(Milpa Alta, siglos XVII-XXI)

de Paula López Caballero

$232-235$

Charlynne Curiel 


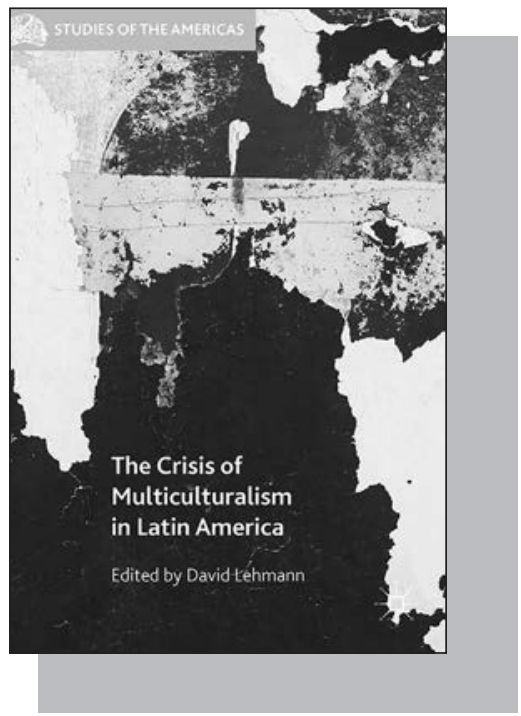

ISSN: 1390-1249

DOI: http://dx.doi.org/10.17141/iconos.61.2018.3386

David Lehmann, editor

The Crisis of Multiculturalism in Latin America

Nueva York: Palgrave Macmillan, 2016, 230 págs.

La crisis del multiculturalismo en América Latina es un texto provocador que, como podrá atestiguarse, amerita más de una lectura. La obra, puesta en circulación en noviembre de 2016, es resultado del trabajo de edición a cargo de David Lehmann ${ }^{1}$ con el respaldo del Instituto de las Américas en la University College London. Confluyen en este trabajo nueve aportaciones, que incluyen el prólogo a cargo de John Gledhill y la introducción por David Lehmann, quienes proponen un rápido recorrido por los siete capítulos en los que se da

1 Desde la década de 1970, el socio-antropólogo se ha interesado por los procesos de transformación social, económica y política en América Latina, entre ellos, la tensa relación entre Estado, estructura agraria, campesinado, democracia y desarrollo. Durante los últimos 20 ańos, indaga sus derivas multiculturales e interculturales en México y Brasil. cuenta de las consecuencias positivas, negativas y también ambiguas del multiculturalismo en regiones americanas de habla hispana y portuguesa ajenas al mundo anglosajón. ${ }^{2}$ Así, la noción de multiculturalismo puesta en circulación en esta obra trabaja alrededor de un conjunto heterogéneo de experiencias colectivas que adquieren un complejo carácter conceptual en la medida en que la retórica jurídica y la práctica diaria de los sujetos de conocimiento son contrastadas críticamente. En ese entramado, el multiculturalismo es susceptible de caracterizarse como:

a) Una política universal, social, económica, ambiental, expresada regional y localmente, y manifiesta en las prácticas de los colectivos humanos. Produce resultados sorpresivos y paradójicos: a pesar de su alcance universal y cobijar reivindicaciones indígenas ligadas con los usos y costumbres, desconoce demandas de equidad de mujeres indígenas como pertinentes al carácter de tales pueblos.

b) Convergencia entre estructura jurídico-administrativa del Estado, sociedad civil organizada, agencias globales de desarrollo, capital y, por supuesto, pueblos indios (fundamentalmente autorreconocidos, nombrados "desde afuera" o etno-estratégicamente situados en el marco de coyunturas particulares, políticas y económicas). Convergencia orientada a emitir

2 La secuencia de los textos responde al siguiente orden: "El multiculturalismo como arma jurídica: uso y abuso del concepto de "pueblo originario" en los conflictos agrarios en Michoacán, México", a cargo de Luis Vázquez León; "Las paradojas del multiculturalismo en Bolivia", por Andrew Canessa; "La etnización de los conflictos agrarios: un caso argentino", de Maité Boullosa-Joly; "La invención de nuestros propios derechos: mujeres que trascienden la oposición entre lo indígena y lo universal", con Manuela Lavinas Picq; "La demanda por el reconocimiento y el acceso a la ciudadanía: etiquetamiento étnico y reestructuración territorial en Brasil", autoría de Véronique Boyer; y "La política de nombrar: acción afirmativa en la educación superior brasileña”, por el propio David Lehmann. 
reivindicaciones al amparo de la academia o la intelectualidad -y también desde su franca oposición-, provocando efectos que suelen salirse del control estatal, como las medidas estatales de restitución de tierras.

c) Imbricación con el capitalismo neoliberal a guisa de ideología, no siempre transparente al análisis en los reclamos vinculados con la resolución de juicios agrarios de larga data. Asignación de tierras que transforman la posesión colectiva en privada y la insertan en circuitos económicos de alta rentabilidad, explotación, despojo y reasignación territorial de población sobre la base de identidades pretéritas, recientemente inventadas.

d) Potenciación de la reestructuración neoliberal de los mercados de trabajo, reconfigurando el equilibrio entre capital y trabajo mediante la desregulación o la desorganización del mismo: una de sus expresiones es el trabajo en condiciones de informalidad que propicia relaciones interétnicas e intercomunitarias apoyadas en la violencia administrada por los gobiernos estatales en consenso con autoridades indígenas legitimadas por la propia administración estatal.

e) Conciliación tortuosa del reconocimiento de la diferencia cultural y de la identidad con la redistribución de la riqueza. $\mathrm{Al}$ señalar que la esfera de la superestructura es autónoma e independiente de las relaciones de producción y reproducción, constriñe las demandas de los grupos a determinantes unívocos y excluyentes: el color de la piel, la lengua, la sexualidad, el género, pero no las relaciones de producción y reproducción.

f) Promoción exacerbada de afectos hacia los pueblos indios y tendencia a la mitificación de sus prácticas económicas y relaciones sociales de propiedad. En tanto se dice que éstos encarnan formas alternativas de aprehender el mundo -lo cual, por otra parte, es también resultado de un fenómeno de exotización de la alteridad-, suele derivarse la afirmación de que en los pueblos originarios la existencia individual solo es posible en un tono comunal y las motivaciones subjetivas no subyacen al ejercicio de poder o a formas de dominio comunitario.

Con la emergencia y reconocimiento internacional de los derechos de los pueblos indígenas y tribales proclamado en el Convenio 169 de la Organización Internacional del Trabajo (OIT) en 1989, se expandió por el orbe la sensación de conciencia étnica y la consecuente politización de la raza y la etnicidad. Lo que en el derecho positivo consistió en la aceptación de las diferencias culturales por parte de los Estados nacionales firmantes del Convenio, ${ }^{3}$ en diversas experiencias latinoamericanas se plasmó como la emergencia de relaciones de poder productoras de nuevas desigualdades e injusticias sociales en torno al acceso al territorio y al derecho a regular la vida social de un modo particular. Fenómeno cobijado por la política del reconocimiento, una posición de poder para determinar qué es auténtico, qué expresión da cuenta de la pertenencia identitaria, quién puede o no disponer de los atributos necesarios para calificar como pueblo originario poseedor de usos y costumbres, y ser tratado de un modo singular.

Para Lehmann, la noción de multiculturalismo pone en entredicho la supuesta apertura de las sociedades latinoamericanas a la diferencia racial, étnica, de clase, de género, entre otras, y en cambio hace florecer su contraparte: la interculturalidad. Ésta consistiría en ofertas de reconocimiento e institucionalización de medidas educativas y jurídicas dis-

3 Los gobiernos de Cuba y la República Oriental del Uruguay constituyen la excepción en América Latina. 
tintivas adaptadas a la herencia o cultura de pueblos indígenas (nominadas como usos y costumbres), a un perfil público de "indigenidad" como categoría que cubre afiliaciones lingüísticas, territoriales y culturales múltiples, así como políticas integracionistas diseñadas expresamente para los pueblos indígenas, para permitirles participar en términos iguales en la cultura hegemónica, en la educación y en los mercados de trabajo (p. 4).

En continuidad con lo señalado, una de las bases en que se apoya la política insuficiente del multiculturalismo es su señalamiento incisivo sobre las dicotomías falsas asociadas con lo indígena: es habitual pensar que las variaciones culturales devienen de diferencias étnicas o lingüísticas y no son producto de diferencias en el orden socioeconómico, ecológico, regional y religioso, es decir, suele predominar la idea esencialista que categoriza a los pueblos indios por su distinción cultural. Otra dicotomía falsa se resume en la idea de los sistemas jurídicos como propios de la regulación de los Estados modernos y ausentes en los marcos regulatorios de los pueblos indios, en cuyo caso son adjetivados como "justicia indígena”, "justicia comunitaria”, "justicia popular" y "pluralismo legal" (p. 6). Sin embargo, estudios de caso en Guatemala, Ecuador y México (como el alzamiento zapatista en este último) muestran que la disputa no se refiere a la reivindicación del acceso, al control y decisión sobre los recursos del territorio, como tampoco a una demanda asociada con la singularidad cultural indígena, sino a la exigencia a la autoridad judicial de la aplicación de la justicia social según lo establece la ley en términos administrativos.

En esas circunstancias, "pasar por indígena" o ser indígena resulta una estrategia coyuntural de acción colectiva que busca acceder a la impartición de justicia en condiciones de equidad; $y$ hacer esto irremedia- blemente sitúa las movilizaciones sociales o auténticamente indígenas en un marco de jurisprudencia de alcance universal, que supondría su desencialización.

Los afectos de los urbanitas -académicos, sociedad civil organizada, agencias nacionales e internacionales de desarrollo- hacia el repudio del coloniaje histórico cebado en lo indígena, suelen poner los temas de la justicia, la democracia, el reconocimiento y la distribución de la riqueza en un precario equilibrio que, en no pocas ocasiones, conduce a la manifestación de injusticias que no subsanan las primeras y generan nuevas dinámicas basadas en la tensión, el conflicto y la ambigüedad. En nombre de la identidad, se omite afirmar que ésta es una construcción social para sustituirla por una tenue línea de corte esencialista. En toda esta discusión, al final el papel del Estado es relevante para garantizar su propia capacidad de determinar la corrección de las reivindicaciones bajo el sino indígena; es decir, el Estado legitima y legaliza el reconocimiento a la identidad. A decir de Lehmann, resulta irónico que, para restaurar la tenencia agraria indígena, sea necesario movilizar el régimen de propiedad estatal con todo su aparato de clasificación, medición y regulación territorial que choca con muchas formas indígenas de tenencia y puede estar muy mal adaptada a los modos predominantes de producción y reproducción (p. 13).

La idea de "indigenidad", es decir, el grado por el cual sería posible determinar el nivel de autenticidad de grupos de individuos singulares, subyace a la discusión sociopolítica latinoamericana cuyo correlato es la promoción discursiva y práctica de niveles variables de legalidad y legitimidad en estos grupos para ganar acceso, control y decisión sobre recursos territoriales y formas productivas y reproductivas allí inscritas. Esta promoción, caracterizada por Gledhill como "técnicas neoliberales 
de gobierno", refiere expresiones de gubernamentalidad neoliberal que desempoderan movimientos indígenas, promueven sujetos indígenas legítimos e impulsan desde las élites locales contramovimientos sociales. Esto, en aras del reconocimiento de la igualdad y, sobre todo, de las contribuciones exclusivas de los pueblos indígenas y tribales a la "armonía social y ecológica de la humanidad" (p. viii) para presentar tales aportaciones, afectivamente muy valoradas, como alternativas al modelo de desarrollo económico.

Sin embargo, tiene su contrasentido en la propia declaración 169 de la OIT, cuando se reconoce el derecho de los Estados o de las concesiones asignadas por los Estados a las sociedades anónimas a explotar minerales y otros recursos del subsuelo, al tiempo que se establecen procedimientos mediante los cuales los pueblos indígenas pueden enajenar sus tierras o transmitir sus derechos fuera de su comunidad (p. viii). El ejemplo argentino respecto a la etnogénesis, potenciado por la estructura de oportunidad política de la mea culpa histórica del Gobierno argentino, da cuenta de esa recurrencia a la identidad como señal de autenticidad, que, en este caso, se descubre como fenómeno de inautenticidad. Cuando trabajadores rurales buscan pasar como herederos de pueblos indígenas -tal como ocurre en Michoacán con mayor éxito para grupos particulares, y como reiteradamente insiste Luis Vázquez León-, llevando a la disyuntiva en el ejercicio jurídico estatal por el decantamiento hacia la (in)justicia de algunos pueblos y/o el bienestar de otros. Paralelamente, la indigenidad, sobre la base de una aceptada e idealizada homogeneidad, supone como, en el caso boliviano, la producción organizada de lo indígena que, apelando al fundamento de los usos y costumbres, induce al diseño territorializado y desterritorializado de pueblos indígenas. Es decir, induce a la implantación de una identidad que no tiene conexión necesaria con formas de vida ancestrales pero que logra, de manera efectiva, interpelación con poblaciones urbanas sensibles a conceder derechos a los pueblos originarios.

Para hacer extensiva la invitación a leer La crisis del multiculturalismo en América Latina, quiero referir el proverbial regaño, narrado por John Gledhill en el prólogo, que condensa la complejidad del rumbo que los afectos nostálgicos exacerbados toman entre la comunidad académica mexicana -probablemente ello también ocurra en otras latitudes latinoamericanas- y que, según sus propias palabras, le permite afirmar que ella tiende a estar particularmente nerviosa con respecto al esencialismo estratégico y las tradiciones inventadas (p. vi). Me pregunto si ese nerviosismo en torno al esencialismo estratégico y las tradiciones inventadas es similar a la denominación de origen de ciertos productos emblemáticos de la identidad mexicana como la bebida alcohólica mezcal: la parte bella del tema es que son únicos, auténticos y originales; la parte bestial es que, al eternizarlos, los hacemos inmanentes y les negamos el derecho al cambio.

Luis Fernando Gutiérrez Dominguez Universidad Autónoma de Puebla, México 